\title{
ON PAIRS OF COMMUTING NILPOTENT MATRICES
}

\author{
TOMAŽ KOŠIR AND POLONA OBLAK
}

\begin{abstract}
Let $B$ be a nilpotent matrix and suppose that its Jordan canonical form is determined by a partition $\lambda$. Then it is known that its nilpotent commutator $\mathcal{N}_{B}$ is an irreducible variety and that there is a unique partition $\mu$ such that the intersection of the orbit of nilpotent matrices corresponding to $\mu$ with $\mathcal{N}_{B}$ is dense in $\mathcal{N}_{B}$. We prove that map $\mathcal{D}$ given by $\mathcal{D}(\lambda)=\mu$ is an idempotent map. This answers a question of Basili and Iarrobino [9] and gives a partial answer to a question of Panyushev 18. In the proof, we use the fact that for a generic matrix $A \in \mathcal{N}_{B}$ the algebra generated by $A$ and $B$ is a Gorenstein algebra. Thus, a generic pair of commuting nilpotent matrices generates a Gorenstein algebra. We also describe $\mathcal{D}(\lambda)$ in terms of $\lambda$ if $\mathcal{D}(\lambda)$ has at most two parts.
\end{abstract}

\section{INTRODUCTION}

We denote by $M_{n}(F)$ the algebra of all $n \times n$ matrices over an algebraically closed field $F$ and by $\mathcal{N}$ the variety of all nilpotent matrices in $M_{n}(F)$. Let $B \in \mathcal{N}$ be a nilpotent matrix and suppose that its Jordan canonical form is determined by a partition $\lambda$. We denote by $\mathcal{O}_{B}=\mathcal{O}_{\lambda}$ the orbit of $B$ under the $\mathrm{GL}_{n}(F)$ action on $\mathcal{N}$ and by $\mathcal{N}_{B}$ the nilpotent commutator of $B$, i.e. the set of all $A \in \mathcal{N}$ such that $A B=B A$. It is known that $\mathcal{N}_{B}$ is an irreducible variety (see Basili [2]). So there is a unique partition $\mu$ of $n$ such that $\mathcal{O}_{\mu} \cap \mathcal{N}_{B}$ is dense in $\mathcal{N}_{B}$. Following Basili and Iarrobino [9], and Panyushev [18] we define a map on the set of all partitions of $n$ by $\mathcal{D}(\lambda)=\mu$. (We note that in [3, 9] this map is denoted by $Q$.) As in [18] we say that $B$ or its orbit $\mathcal{O}_{\lambda}$ is self-large if $\mathcal{D}(\lambda)=\lambda$. According to [3, 9], a partition $\lambda$ is called stable if $\mathcal{D}(\lambda)=\lambda$.

The work presented in this paper was initially stimulated by a question if the partition $\mathcal{D}(\lambda)$ is stable, i.e. if $\mathcal{D}$ is an idempotent map, that was posed by Basili and Iarrobino in their conference notes [9]. It was later brought to our attention that Panyushev in [18, Problem 2] stated the same question in a more general setup of simple Lie algebras. The general question in the theory of nilpotent orbits of semisimple Lie algebras is similar to the question described above. Suppose that $\mathfrak{g}$ is a semisimple Lie algebra and $G$ its adjoint group. If $x \in \mathfrak{g}$ is a nilpotent element and $\mathfrak{z}_{\mathfrak{g}}(x)$ its centralizer in $\mathfrak{g}$, then there is a unique maximal nilpotent $G$-orbit, say $G y$, meeting $\mathfrak{z}_{\mathfrak{g}}(x)$. It was pointed out to us by a referee that the latter follows from the fact that the set $\mathcal{N}_{\mathfrak{h}}$ of all nilpotent elements of an algebraic Lie algebra $\mathfrak{h}$ is irreducible, which in turn can be deduced from the existence of the Levi decomposition and the irreducibility of $\mathcal{N}_{\mathfrak{h}}$ in the reductive case, which was proved already by Kostant [10, §3]. The question is then if the largest nilpotent orbit meeting $\mathfrak{z} \mathfrak{g}(y)$ is $G y$ itself [18, Problem 2]. A nilpotent orbit $G y$ is said to be self-large, if

Date: August 14, 2021.

2000 Mathematics Subject Classification. Primary. 13E10, 15A27. Secondary. 14L30.

Key words and phrases. Nilpotent matrices, commuting matrices, nilpotent commutator, nilpotent orbit, maximal partition, Gorenstein artinian algebra. 
it is the largest nilpotent orbit meeting $\mathfrak{z} \mathfrak{g}(y)$. Thus, the question is if the largest nilpotent orbit meeting $\mathfrak{z} \mathfrak{g}(x)$ is necessarily self-large.

Our main result is the proof of the fact that $\mathcal{D}$ is an idempotent map on nilpotent orbits of $M_{n}(F)$. This answers the original question of Basili and Iarrobino and hence also a special $\mathfrak{s} l_{n}$ case of Panyushev's question. In the proof we use an extension of a lemma of Baranovsky [1]. We prove that a generic pair of commuting nilpotent matrices generates a Gorenstein algebra (in fact, a complete intersection [5, Cor. 21.20]). Moreover, a generic matrix $A \in \mathcal{N}_{B}$ and $B$ generate a Gorenstein algebra. Then we use Macaulay's Theorem on the Hilbert function of the intersection of two plane curves [12] (see also [8]) together with some results of 3 to prove that $\mathcal{D}$ is an idempotent. It appears that an answer to the general question posed by Panyushev [18, Problem 2] requires methods different from ours. We see no immediate generalization of our proof to the general setup of simple Lie algebras.

It is an interesting question [18, Problem 1] to describe $\mathcal{D}(\lambda)$ in terms of partition $\lambda$. We would like to mention that some partial results to this question were obtained by the second author in [14, 15]. The main result of [14] (see also [4]) gives the answer in the case when $\mathcal{D}(\lambda)$ has at most two parts. We discuss this in the last section.

Panyushev's work [18] was motivated by Premet's results on the nilpotent commuting variety [21] of a simple Lie algebra. For other results on special pairs of nilpotent commuting elements in these varieties see [6, 17]. Some of other references for the theory of commuting varieties are [16, 19, 20, 22, 23].

This paper is an extension of our unpublished note [11].

\section{Gorenstein PAirs ARE Dense}

In this section we prove that a generic pair of commuting nilpotent matrices generates a Gorenstein (local artinian) algebra.

Suppose that $B \in \mathcal{N}$ and that $A \in \mathcal{N}_{B}$. Then we denote by $\mathcal{A}=\mathcal{A}(A, B)$ the unital subalgebra of $M_{n}(F)$ generated by matrices $A$ and $B$, and by $\mathcal{A}^{T}=\mathcal{A}\left(A^{T}, B^{T}\right)$ the unital subalgebra generated by the transposed matrices $A^{T}$ and $B^{T}$. The algebra $\mathcal{A}$ is a commutative local artinian algebra. Such an algebra is Gorenstein if its $\operatorname{socle} \operatorname{Soc}(\mathcal{A})$ is a simple $\mathcal{A}$-module (see [5, Prop. 21.5]); i.e., if $\operatorname{dim}_{F}(\operatorname{Soc}(\mathcal{A}))=1$, where $\operatorname{Soc}(\mathcal{A})=(0: m)$ is the annihilator of the maximal ideal $m$ of $\mathcal{A}$.

We write $\mathcal{N}_{2} \subset M_{n}(F) \times M_{n}(F)$ for the variety of all commuting pairs of nilpotent matrices. Note that the subset $U \subset \mathcal{N}_{2} \times F^{n} \times F^{n}$ of all quadruples $(A, B, v, w)$ such that $v$ is a cyclic vector for $(A, B) \in \mathcal{N}_{2}$ and $w$ cyclic for $\left(A^{T}, B^{T}\right)$ is an open subset. The fact that $U$ is open follows since its complement is given by a set of polynomial conditions $\operatorname{det} X=0$ and $\operatorname{det} Y=0$, where $X$ runs over all square matrices with columns of the form $A^{i} B^{j} v$ and $Y$ over all square matrices with the columns of the form $\left(A^{T}\right)^{i}\left(B^{T}\right)^{j} w$. Here it is certainly enough to take $0 \leq i, j \leq n-1$. The same argument shows that also $U_{B}=\{(A, v, w) ;(A, B, v, w) \in U\}$ is an open subset of $\mathcal{N}_{B} \times F^{n} \times F^{n}$.

Lemma 2.1. Suppose that $(A, B) \in \mathcal{N}_{2}$. Then there is a third nilpotent matrix $C$ and vectors $v, w \in F^{n}$ such that:

(i) $C$ commutes with $B$,

(ii) any linear combination $\alpha A+\beta C$ is nilpotent,

(iii) $v$ is a cyclic vector for $(C, B)$, 
(iv) $w$ is a cyclic vector for $\left(C^{T}, B^{T}\right)$.

Proof. The proof is an extension of the proof of Baranovsky [1, Lem. 3]. Let $\lambda=$ $\left(\lambda_{1}^{r_{1}}, \lambda_{2}^{r_{2}} \ldots, \lambda_{l}^{r_{l}}\right)$, where $\lambda_{1}>\lambda_{1}>\cdots>\lambda_{l}>0$ and $r_{i} \geq 1$ for all $i$ be the partition corresponding to $B$. As in the proof of [1, Lem. 3] there is a Jordan basis

$$
\left\{e_{i j k}: 1 \leq i \leq l, 1 \leq j \leq r_{i}, 1 \leq k \leq \lambda_{i}\right\}
$$

for $B$ such that

(1) $B e_{i j k}=e_{i j, k+1}$ if $k<\lambda_{i}$ and $B e_{i j \lambda_{i}}=0$,

(2) $A e_{i j k}$ is in the linear span of vectors $e_{f g h}$, where either

- $f>i$ and $g, h$ arbitrary, or

- $f=i$ and $g>j$ and $h$ arbitrary, or

- $f=i$ and $g=j$ and $h>k$.

To simplify our expressions we assume that $e_{i j k}=0$ if the three indices $i, j, k$ do not satisfy the conditions $1 \leq i \leq l, 1 \leq j \leq r_{i}$ and $1 \leq k \leq \lambda_{i}$. We also use the difference sequence of $\lambda$; we write $\delta_{i}=\lambda_{i}-\lambda_{i-1}$ for $i=2,3 \ldots, l$. Now we define $C$ by $C e_{i j k}=e_{i, j+1, k}$ if $j<r_{i}$ and $C e_{i, r_{i}, k}=e_{i+1,1, k}+e_{i-1,1, k+\delta_{i}}$.

To illustrate the actions of $B$ and $C$ we include an example. We consider the case $\lambda=\left(4^{2}, 3^{2}, 2,1^{2}\right)$. To indicate the actions of $B$ and $C$ we draw a directed graph whose vertices correspond to the vectors of the Jordan basis $\left\{e_{i j k}\right\}$ and the edges to the nonzero elements of $B$ and $C$. In the graph in Figure 1 the dashed directed edges correspond to

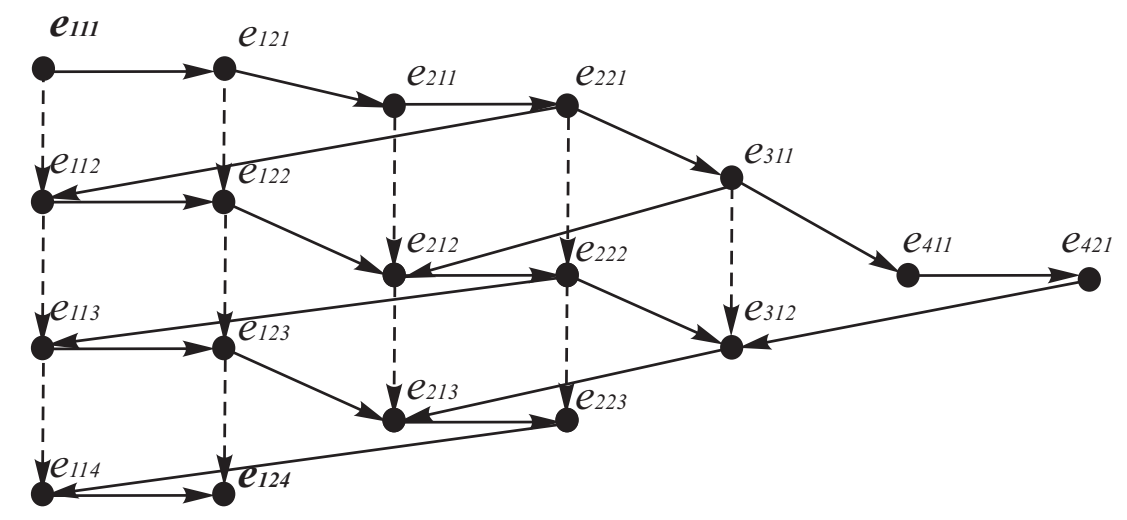

FiguRE 1.

the action of matrix $B$ and the nondashed ones correspond to the action of matrix $C$. Note that the graph indicating the action of $B^{T}$ and $C^{T}$ is obtained by reversing all the edges in the graph corresponding to $B$ and $C$. Note that vector $e_{111}$ is cyclic and vector $e_{124}$ cocyclic, i.e., cyclic for $B^{T}$ and $C^{T}$.

Let us continue with the proof. It is easy to show that $B C=C B$; we prove directly that $B C e_{i j k}=C B e_{i j k}$ for all $i, j, k$. The property (ii) follows from (2) and the definition of $C$.

Next we show that $e_{111}$ is a cyclic vector for $(C, B)$ and $e_{1 r_{1} \lambda_{1}}$ is a cyclic vector for $\left(C^{T}, B^{T}\right)$. We denote by $\mathcal{A}$ the unital subalgebra of $M_{n}(F)$ generated by $B$ and $C$ and by $\mathcal{A}^{T}$ the unital subalgebra generated by the transposed matrices $B^{T}$ and $C^{T}$. We show by induction on $i$ that each $e_{i j k}$ is in the subspace $\mathcal{A} e_{111}$. For $i=1$ we have 
$e_{1 j k}=B^{k-1} C^{j-1} e_{111}$. We denote by $W_{i}$ the linear span of all vectors $e_{t j k}$ with $t \leq i$. To prove the inductive step, we have to show that $e_{i+1, j, k} \in \mathcal{A} W_{i}$. This follows since $e_{i+1, j, k}-B^{k-1} C^{j} e_{i, r_{i}, 1} \in \mathcal{A} W_{i}$.

Before we prove that $e_{1 r_{1} \lambda_{1}}$ is a cyclic vector for $\left(C^{T}, B^{T}\right)$ observe that $B^{T} e_{i j k}=e_{i j, k-1}$ and that $C^{T} e_{i j k}=e_{i, j-1, k}$ if $j>1$ and $C^{T} e_{i 1 k}=e_{i-1, r_{i-1}, k}+e_{i+1, r_{i+1}, k-\delta_{i+1}}$. Again we proceed to prove that $e_{1 r_{1} \lambda_{1}}$ is cyclic by induction on $i$.

For $i=1$ we have $e_{1 j k}=\left(B^{T}\right)^{\lambda_{1}-k}\left(C^{T}\right)^{r_{1}-j} e_{1 r_{1} \lambda_{1}}$. The inductive step follows since $e_{i+1, j, k}-\left(B^{T}\right)^{\lambda_{i}-k}\left(C^{T}\right)^{r_{i}-j} e_{i r_{i} \lambda_{i}} \in \mathcal{A}^{T} W_{i}$.

Proposition 2.2. The subset $U$ is dense in $\mathcal{N}_{2} \times F^{n} \times F^{n}$ and the subset $U_{B}$ is dense in $\mathcal{N}_{B} \times F^{n} \times F^{n}$.

Proof. Consider a quadruple $(A, B, v, w) \in \mathcal{N}_{2} \times F^{n} \times F^{n}$. By Lemma 2.1 we can find a matrix $C$ and vectors $v^{\prime}, w^{\prime}$ such that $\left(C, B, v^{\prime}, w^{\prime}\right) \in U$. Then the affine line $L$ of all the points $\left(\alpha A+\alpha^{\prime} C, B, \alpha v+\alpha^{\prime} v^{\prime}, \alpha w+\alpha^{\prime} w^{\prime}\right)$, where $\alpha \in F$ is arbitrary and $\alpha^{\prime}=1-\alpha$, has nonempty intersection with $U$. Hence $L \cap U$ is dense in $L$ and $(A, B, v, w)$ is in the closure of $U$.

The same argument shows that also $U_{B}$ is dense in $\mathcal{N}_{B} \times F^{n} \times F^{n}$.

Next we give a proof of a known result which we could not find stated in the literature.

Proposition 2.3. A commutative subalgebra $\mathcal{R}$ of $M_{n}(F)$ is Gorenstein if both $\mathcal{R}$ and $\mathcal{R}^{T}$ have a cyclic vector, i.e., the action of $\mathcal{R}$ is cyclic and cocyclic.

Proof. By Lemma 2.5, parts (1) and (2), of [13] the subalgebra $\mathcal{R}$ is cyclic if and only if $F^{n}$ and $\mathcal{R}$ are isomorphic as $\mathcal{R}$-modules, and the subalgebra $\mathcal{R}^{T}$ is cyclic if and only if $F^{n}$ and $\mathcal{R}^{T}$ are isomorphic $\mathcal{R}$-modules. Then our assumptions imply that $\mathcal{R}$ and its dual module are isomorphic $\mathcal{R}$-modules, and thus $\mathcal{R}$ is Gorenstein by the definition [5, pp. 525-526].

We denote by $\pi: \mathcal{N}_{2} \times F^{n} \times F^{n} \rightarrow \mathcal{N}_{2}$ and $\pi_{B}: \mathcal{N}_{B} \times F^{n} \times F^{n} \rightarrow \mathcal{N}_{B}$ the projections to the first factor. Then, it follows by Proposition 2.3 that $\pi(U)$ is the set of pairs $(A, B)$ of nilpotent matrices such that the unital algebra $\mathcal{A}$ generated by $A$ and $B$ is Gorenstein of (vector space) dimension $n$. Moreover, such an $\mathcal{A}$ is a complete intersection since its embedding dimension is at most two [5. Cor. 21.20]. Similarly, $\pi_{B}\left(U_{B}\right)$ is the set of all $A \in \mathcal{N}_{B}$ such that $\mathcal{A}(A, B)$ is Gorenstein of dimension $n$.

As a consequence of Proposition 2.2 we obtain the main results of this section:

Corollary 2.4. The subset $\pi(U)$ of those pairs in $\mathcal{N}_{2}$ that generate a Gorenstein algebra of (vector space) dimension $n$ is dense in $\mathcal{N}_{2}$.

Corollary 2.5. The subset $\pi_{B}\left(U_{B}\right)$ is dense in $\mathcal{N}_{B}$. So, the unital subalgebra $\mathcal{A}$ generated by a nilpotent matrix $B$ and a generic matrix $A$ in $\mathcal{N}_{B}$ is Gorenstein; moreover, it is a complete intersection. Equivalently, both $\mathcal{A}$ and $\mathcal{A}^{T}$ have a cyclic vector, i.e., the action of $\mathcal{A}$ is cyclic and cocyclic.

\section{3. $\mathcal{D}$ IS AN IDEMPOTENT MAP}

A pair of commuting nilpotent matrices $(A, B)$ generates a (unital) local artinian algebra $\mathcal{A}$. We denote its maximal ideal by $m$. The associated graded algebra of $\mathcal{A}$ is

$$
\operatorname{gr} \mathcal{A}=\underset{4}{\oplus_{i=0}^{k}} m^{i} / m^{i+1}
$$


where $m^{0}=\mathcal{A}$ and $k$ is such that $m^{k} \neq 0$ and $m^{k+1}=0$. The Hilbert function $H(\mathcal{A})$ of $\mathcal{A}$ is the sequence $\left(h_{0}, h_{1}, \ldots, h_{k}\right)$, where

$$
h_{i}=\operatorname{dim}_{F} m^{i} / m^{i+1} .
$$

We have $\sum_{i=0}^{k} h_{i}=\operatorname{dim}_{F} \mathcal{A}$. If $\mathcal{A}$ is Gorenstein then $h_{k}=1$. Furthermore, Macaulay's Theorem on the Hilbert function of the intersection of two plane curves 12] (see Iarrobino [7] or [8, p. 23]) says that the Hilbert function $H(\mathcal{A})$ of an artinian local complete intersection of the embedding dimension at most 2 satisfies

$$
H(\mathcal{A})=\left(1,2, \ldots, d, h_{d}, h_{d+1}, \ldots, h_{i}, \ldots, h_{k}\right),
$$

where $h_{d-1}=d \geq h_{d} \geq h_{d+1} \geq \ldots \geq 1$ and $h_{i-1}-h_{i} \leq 1$ for all $i=d, d+1, \ldots, k$ and $h_{k}=1$.

In the rest of the paper, we write $\lambda=\left(\lambda_{1}, \lambda_{2}, \ldots, \lambda_{l}\right)$, where $\lambda_{1} \geq \lambda_{2} \geq \cdots \geq \lambda_{l} \geq 1$. (Compare with the proof of Lemma 2.1, where we used different, 'power type', notation for parts of partition $\lambda$.) We assume that $\lambda_{j}=0$ for $j>l$.

The set of all partitions $\Lambda(n)$ of $n$ has a partial order given by $\lambda \prec \mu$ if $\sum_{i=1}^{j} \lambda_{i} \leq \sum_{i=1}^{j} \mu_{i}$ for $j=1,2, \ldots$. To each partition $\lambda$ we associate its Ferrers diagram, a diagram with $\lambda_{i}$ boxes in the $i$-th row. We denote by $\lambda(H)$ the partition, which has the $i$-th part equal to the number of elements in the sequence $H=\left(h_{1}, h_{2}, \ldots, h_{k}\right)$ such that $h_{j} \geq i$.

Example 3.1. If $H=(1,2,3,2,1)$ then $\lambda(H)=(5,3,1)$ and if $H=(1,2,3,3,1)$ then $\lambda(H)=(5,3,2)$.

Here we recall two results of Basili and Iarrobino [3] that we use in the proof below. They proved [3, Thm. 2.21] that the Hilbert functions of the algebras $\mathcal{A}(A, B), A \in \mathcal{N}_{B}$, determine $\mathcal{D}(\lambda)$ :

$$
\mathcal{D}(\lambda)=\sup \left\{\lambda(H(\mathcal{A})) ; \mathcal{A}=\mathcal{A}(A, B), A \in \mathcal{N}_{B}, \text { such that } \operatorname{dim} \mathcal{A}=n\right\} .
$$

They also proved [3, Thm. 1.12] that if the parts in a partition $\lambda$ differ by at least two then $\lambda$ is stable, i. e. $\mathcal{D}(\lambda)=\lambda$.

The following is the main result of our paper.

Theorem 3.2. Assume that $B$ is a nilpotent matrix and $\lambda$ the corresponding partition. Then the partition $\mathcal{D}(\lambda)$ corresponding to the generic element $A \in \mathcal{N}_{B}$ has decreasing parts differing by at least 2 and $\mathcal{D}(\mathcal{D}(\lambda))=\mathcal{D}(\lambda)$, i.e. $\mathcal{D}$ is idempotent.

Proof. Recall that $\mathcal{D}(\lambda)$ is given by (1). Corollary 2.5 implies that for a generic $A \in \mathcal{N}_{B}$ the algebra $\mathcal{A}=\mathcal{A}(A, B)$ is a complete intersection (therefore Gorenstein) of dimension $n$. Since $\mathcal{N}_{B}$ is irreducible and since $\mathcal{D}(\lambda)$ is the partition corresponding to the generic $A \in \mathcal{N}_{B}$, it is enough to take in (11) the supremum over all $\lambda(H(\mathcal{A})$ ), where $\mathcal{A}$ is a complete intersection. The above stated Macaulay's Theorem on the Hilbert function of the intersection of two plane curves then implies that the parts in $\mathcal{D}(\lambda)$ differ by at least two. By [3, Thm. 1.12] or [9, Cor 1.5] it follows that $\mathcal{D}(\mathcal{D}(\lambda))=\mathcal{D}(\lambda)$.

\section{Description of $\mathcal{D}(\lambda)$ When it has at most two parts}

A partition $\lambda$ is called a almost rectangular if the largest and the smallest part of $\lambda$ differ by at most one. (Note that in [3] the term string is used for almost rectangular partition.) We denote by $r_{B}$ or $r_{\lambda}$ the smallest number $r$ such that $\lambda$ is a union of $r$ almost 
rectangular partitions. (Here $B$ is a nilpotent matrix such that its Jordan canonical form is determined by $\lambda$.) Basili [2, Prop. 2.4] proved that a generic $A \in \mathcal{N}_{B}$ has rank $n-r_{B}$, which is maximal possible in $\mathcal{N}_{B}$ and that $\mathcal{D}(\lambda)$ has $r_{B}$ parts.

Example 4.1. For instance, if $\lambda=(4,3,2,1)$ and $\mu=(7,7,6,4,4,3,2)$ then $r_{\lambda}=2$ and $r_{\mu}=3$.

It is an interesting and important question to describe $\mathcal{D}(\lambda)$ in terms of $\lambda$ (see [18, §3], in particular [18, Problem 1]). Using [14, Thm. 13] and [4, Cor. 3.29] it is easy to answer this question when $\mathcal{D}(\lambda)$ has at most two parts, i. e. when $r_{B} \leq 2$. Here we would like to remark that the proofs of Lemma 11 and Theorems 12 and 13 in [14 hold over any field of characteristic 0, while Basili and Iarrobino assume in [4] that the underlying field is algebraically closed of arbitrary characteristic.

Now, if $r_{B}=1$ then $\mathcal{D}(\lambda)=(n)$. If $r_{B}=2$ then it is enough to know the maximal index of nilpotency of an element of $\mathcal{N}_{B}$ to describe $\mathcal{D}(\lambda)$, since $\mathcal{D}(\lambda)$ is the maximal partition among those corresponding to elements $A \in \mathcal{N}_{B}$ [3, Lem. 1.6]. Applying the description of the maximal index of nilpotency given in [14, Thm. 13] and [4, Cor. 3.29], we have the following result.

Theorem 4.2. If $\lambda=\left(\lambda_{1}, \lambda_{2}, \ldots, \lambda_{l}\right), \lambda_{1} \geq \lambda_{2} \geq \ldots \geq \lambda_{l} \geq 1$ is such that $r_{\lambda}=2$ then $\mathcal{D}(\lambda)=\left(i_{\lambda}, n-i_{\lambda}\right)$, where $i_{\lambda}$ is the maximal index of nilpotency in $\mathcal{N}_{B}$ given by

$$
i_{\lambda}=\max _{1 \leq i<l}\left\{2(i-1)+\lambda_{i}+\lambda_{i+1}+\ldots+\lambda_{i+r} ; \lambda_{i}-\lambda_{i+r} \leq 1, \lambda_{i-1} \geq 2 \text { if } i>1\right\} .
$$

Example 4.3. Suppose that $\lambda=(4,4,3,3,2)$ and $\mu=(5,5,3,3,2)$. Then we have $\mathcal{D}(\lambda)=$ $(14,2)$ and $\mathcal{D}(\mu)=(12,6)$.

\section{ACKNOWLEDGEMENT}

The authors would like to thank Anthony Iarrobino for stimulating comments that improved the quality of the paper. We would also like to thank him and Roberta Basili

for sharing with us early versions of their papers. We acknowledge financial support of the Research Agency of the Republic of Slovenia.

\section{REFERENCES}

[1] V. Baranovsky. The variety of pairs of commuting nilpotent matrices is irreducible. Transform. Groups 6 (2001), No. 1, 3-8.

[2] R. Basili. On the irreducibility of commuting varieties of nilpotent matrices. J. Algebra 268 (2003), No. 1, 58-80.

[3] R. Basili and A. Iarrobino. Pairs of commuting nilpotent matrices, and Hilbert function. In press, J. Algebra (2008), doi:10.1016/j.jalgebra.2008.03.006

[4] R. Basili and A. Iarrobino. An involution on $\mathcal{N}_{B}$, the nilpotent commutator of a nilpotent Jordan matrix B. Preprint, Aug. 2007.

[5] D. Eisenbud. Commutative Algebra. With a View toward Algebraic Geometry. Springer-Verlag, Berlin, New York, 1995.

[6] V. Ginzburg. Principal nilpotent pairs in a semisimple Lie algebra 1. Invent. Math. 140 (2000), $511-561$.

[7] A. Iarrobino. Punctual Hilbert scheme. Memoirs Amer. Math. Soc. 10 (1977), No. 188.

[8] A. Iarrobino. Associated graded algebra of a Gorenstein Artin algebra. Memoirs Amer. Math. Soc. 107 (1994), No. 514. 
[9] A. Iarrobino jointly with R. Basili. Pairs of commuting nilpotent matrices and Hilbert functions. Extended notes from a talk at the workshop "Algebraic Combinatorics Meets Inverse Systems", Montréal, Jan. 19-21, 2007.

[10] B. Kostant. Lie group representations on polynomial rings. Amer. J. Math. 85 (1963), 327404.

[11] T. Košir and P. Oblak. Note on commuting pairs of nilpotent matrices. Preprint, Feb. 2007.

[12] F. H. S. Macaulay. On a method for dealing with intersections of plane curves. Trans. Amer. Math. Soc. 5 (1904), 385-410.

[13] M. G. Neubauer and D. J. Saltman. Two-generated commutative subalgebras of $M_{n}(F)$. J. Algebra 164 (1994), 545-562.

[14] P. Oblak. The upper bound for the index of nilpotency for a matrix commuting with a given nilpotent matrix. Lin. Multilin. Alg., forthcoming articles, doi: 10.1080/03081080701571083. (See also slightly revised version with more examples on arXiv:math [math.AC:0701561] at http://arxiv.org )

[15] P. Oblak. Jordan forms for mutually annihilating nilpotent pairs. Linear Algebra Appl. 428 (2008), 1476-1491.

[16] D. I. Panyushev. The Jacobian module of a representation of a Lie algebra and geometry of commuting varieties. Compositio Math. 94 (1994), 181-199.

[17] D. I. Panyushev. Nilpotent pairs, dual pairs, and sheets. J. Algebra 240 (2001), 635-664.

[18] D. I. Panyushev. Two results on centralisers of nilpotent elements. J. Pure Appl. Algebra 212 (2008), 774-779.

[19] D. I. Panyushev and O. Yakimova. Symmetric pairs and associated commuting varieties. Math. Proc.Camb. Phil. Soc. 143 (2007), 307-321.

[20] V. L. Popov. Irregular and singular loci of commuting varieties, in press, Transformation Groups 13 (2008), Nos. 3-4, the special issue commemorating Kostant's 80th birthday.

[21] A. Premet. Nilpotent commuting varieties of reductive Lie algebras. Invent. Math. 154 (2003), 653-683.

[22] R. Richardson. Commuting varieties of semisimple Lie algebras and algebraic groups. Compositio Math. 38 (1979), 311-327.

[23] W. V. Vasconcelos. Arithmetic of Blowup Algebras. Cambridge Univ. Press, London Math. Soc. Lect. Note Ser. 195, 1994.

T. Košir: Department of Mathematics, Faculty of Mathematics and Physics, University of Ljubluana, Jadranska 19, SI-1000 Ljubljana, Slovenia; E-Mail: tomaz.kosir@fmF.uni-Lj.Si.

P. Oblak: Department of Mathematics, Institute of Mathematics, Physics, and Mechanics, Jadranska 19, SI-1000 LJubluana, Slovenia; E-Mail: POlOna.oblak@FmF.Uni-LJ.Si.

Current address: University of Ljubljana, Faculty of Computer and Information Science, TrŽaška Cesta 25, SI-1001 Ljubluana, Slovenia. 